\title{
Thermal properties of high density polyethylene-kenaf cellulose composites.
}

\begin{abstract}
Biocomposites of high density polyethylene (HDPE) and kenaf cellulose (KC) with polyethylene glycol (PEG) have been prepared by melt blending and compression moulding. The optimum temperature and reaction period for blending KC with HDPE were $145 \mathrm{C}$ and 12 minutes with rotation speed of $30 \mathrm{rpm}$, respectively. The effects of composition on the thermal properties of HDPE-KC biocomposites were investigated using TGA and DSC. Each sample of HDPE-KC biocomposites was subjected to heating programs between 35-600 C for TGA, and the DSC analysis was determined under heating cycles of 25-600 C. The results from TGA and DSC indicated that by increasing the $\mathrm{KC}$ content, the thermal stability was slightly improved. The melting temperature for all the composites was less than the melting point of $\mathrm{KC}(148.70 \mathrm{C})$, and almost the same as that of HDPE $(130.38 \mathrm{C})$. Therefore no significant change in the melting temperature resulted from adding $\mathrm{KC}$ to the HDPE matrix. However, HDPE's presence surrounding the KC fibres, prevented water from reaching the cellulose fibre particles, thus reducing the water content in the composite; hence a significant advantage of forming $\mathrm{KC}$ composites with hydrophobic HDPE is that it helps to make the cellulose hydrophobic instead of hydrophilic.
\end{abstract}

Keyword: Biocomposites; High density polyethylene; Kenaf; Thermal properties. 